\title{
Description of Nuclear Structure Effects in Subbarrier Fusion by the Interacting Boson Model
}

\author{
A.B. Balantekin \\ Department of Physics, University of Wisconsin, \\ Madison, Wisconsin 53706 USA \\ and \\ S. Kuyucak \\ Department of Theoretical Physics, Research School of Physical Sciences, \\ Australian National University, Canberra, ACT 0200 Australia
}

\begin{abstract}
Recent theoretical developments in using the Interacting Boson Model to describe nuclear structure effects in fusion reactions below the Coulomb barrier are reviewed. Methods dealing with linear and all orders coupling between the nuclear excitations and the translational motion are discussed, and the latter is found to lead to a better description of the barrier distribution data. A systematic study of the available data (cross sections, barrier and spin distributions) in rare-earth nuclei is presented.
\end{abstract}

\section{Introduction}

It is by now well established that to describe fusion cross sections one needs to include coupling of relative motion to other degrees of freedom [1, 2]. These extra degrees of freedom yield a distribution of barriers [3] and consequently enhance the cross section below the barrier [4, 5]. Enhancement of fusion cross sections due to coupling of levels in colliding nuclei to the relative motion, has opened a new avenue for testing nuclear structure models. The recent experimental determination of average angular momenta [6] and the barrier distributions [7 have provided even more stringent tests for such models.

The natural language to study multidimensional barrier penetration is the coupled channels formalism, which was widely used in investigating subbarrier fusion phenomena [1]. An alternative formulation of the multidimensional quantum tunneling is given by the path integral formalism 《⿶. An algebraic nuclear structure model significantly simplifies evaluation of the path integral. The Interacting Boson Model (IBM) of Arima and Iachello [B] is one such model which has been successfully employed to describe the properties of low-lying collective states in medium heavy 
nuclei. Here attempts to use IBM in describing nuclear structure effects in subbarrier fusion are reviewed.

Path integral formulation of this problem, as sketched in the next section, requires analytic solutions for the nuclear wave functions. In our first attempt in using IBM to describe nuclear structure effects in subbarrier fusion, we employed the $\mathrm{SU}(3)$ limit of IBM [9]. However, the SU(3) limit corresponds to a rigid nucleus with a particular quadrupole deformation and no hexadecapole deformation, a situation which is not realized in most deformed nuclei. Thus analytic solutions away from the limiting symmetries of the IBM are needed for realistic calculations of subbarrier fusion cross sections. In a parallel development, a 1/N expansion was investigated [10 for the IBM which provided analytic solutions for a general Hamiltonian with arbitrary kinds of bosons. This technique proved useful in a variety of nuclear structure problems where direct numerical calculations are prohibitively difficult. As we briefly describe in the next section, using the $1 / \mathrm{N}$ expansion technique in the path integral formulation of the fusion problem [11] makes it possible to go away from the three symmetry limits of IBM, in particular, arbitrary quadrupole and hexadecapole couplings can be introduced.

Recently a series of recent high-precision measurements were carried out at the Australian National University (ANU) where distributions of fusion barriers were determined directly from the fusion data [7]. IBM based analysis of fusion cross sections describes the ANU data well [11, 12, 13, 14], especially when the higher order coupling effects are included.

In the next section, first the influence functional method is summarized and its application to the linear coupling of translational motion to the structure of target nuclei is described. The significance of nonlinear couplings is discussed next, and a Green's function method is introduced to describe such nonlinear effects. Section 3 includes attempts to describe subbarrier fusion data with these techniques.

\section{Algebraic Models in Subbarrier Fusion}

The Hamiltonian for the multi-dimensional quantum tunneling problem relevant to subbarrier fusion is

$$
H=-\frac{\hbar^{2}}{2 \mu} \nabla^{2}+H_{0}(\xi)+H_{\mathrm{int}}(\mathbf{r}, \xi)
$$

where $\mathbf{r}$ is the relative coordinate of the target and projectile, and $\xi$ represents any internal degrees of freedom of the target. $H_{0}(\xi)$ and $H_{\text {int }}(\mathbf{r}, \xi)$ describe the internal structure of the target nucleus and its coupling to the relative motion, respectively. In Eq. (1), the propagator to go from an initial state characterized by the relative radial coordinate $r_{i}$ (the magnitude of $\mathbf{r}$ ) and internal quantum numbers $n_{i}$ to a final state characterized by $r_{f}$ and $n_{f}$ may be written as

$$
K\left(r_{f}, n_{f}, T ; r_{i}, n_{i}, 0\right)=\int \mathcal{D}[r(t)] e^{i S(r, T) / \hbar}\left\langle n_{f}\left|\hat{U}_{\mathrm{int}}(r(t), T)\right| n_{i}\right\rangle,
$$


where $S(r, T)$ is the action for the translational motion and $\hat{U}_{\text {int }}$ satisfies the differential equation

$$
\begin{aligned}
i \hbar \frac{\partial \hat{U}_{\mathrm{int}}}{\partial t} & =\left[H_{0}+H_{\mathrm{int}}\right] \hat{U}_{\mathrm{int}}, \\
\hat{U}_{\mathrm{int}}(t=0) & =1 .
\end{aligned}
$$

We want to consider the case where $r_{i}$ and $r_{f}$ are on opposite sides of the barrier. In the limit when the initial and final states are far away from the barrier, the transition amplitude is given by the $S$-matrix element, which can be expressed in terms of the propagator as [4]

$$
\begin{aligned}
S_{n_{f}, n_{i}}(E)= & \frac{i}{\hbar} \lim _{\substack{r_{i} \rightarrow \infty \\
r_{f} \rightarrow-\infty}}\left(\frac{p_{i} p_{f}}{\mu^{2}}\right)^{1 / 2} \exp \left[i\left(p_{f} r_{f}-p_{i} r_{i}\right) / \hbar\right] \\
& \times \int_{0}^{\infty} d T e^{+i E T / \hbar} K\left(r_{f}, n_{f}, T ; r_{i}, n_{i}, 0\right),
\end{aligned}
$$

where $p_{i}$ and $p_{f}$ are the classical momenta associated with $r_{i}$ and $r_{f}$. In heavy ion fusion we are interested in the transition probability in which the internal system emerges in any final state. For the $\ell$ th partial wave, this is

$$
T_{\ell}=\sum_{n_{f}}\left|S_{n_{f}, n_{i}}(E)\right|^{2}
$$

which becomes, upon substituting Eqs. (2) and (5),

$$
\begin{aligned}
T_{\ell}= & \lim _{\substack{r_{i} \rightarrow \infty \\
r_{f} \rightarrow-\infty}}\left(\frac{p_{i} p_{f}}{\mu^{2}}\right) \int_{0}^{\infty} d T e^{i E T / \hbar} \int_{0}^{\infty} \tilde{T} e^{-i E \tilde{T} / \hbar} \\
& \int \mathcal{D}[r(t)] \int \mathcal{D}[\tilde{r}(\tilde{t})] e^{i[S(r, T)-S(\tilde{r}, \tilde{T})] / \hbar} \rho_{M}(\tilde{r}(\tilde{t}), \tilde{T} ; r(t), T) .
\end{aligned}
$$

Here we have assumed that the energy dissipated to the internal system is small compared to the total energy and taken $p_{f}$ outside the sum over final states. We have also identified the two-time influence functional in Eq. (7) as

$$
\rho_{M}(\tilde{r}(\tilde{t}), \tilde{T} ; r(t), T)=\left\langle n_{i}\left|\hat{U}_{\text {int }}^{\dagger}(\tilde{r}(\tilde{t}), \tilde{T}) \hat{U}_{\text {int }}(r(t), T)\right| n_{i}\right\rangle
$$

where the completeness of final states was used. Eq. (8) displays the utility of the influence functional method when the internal system has symmetry properties. If the Hamiltonian in Eq. (3) has a dynamical or spectrum generating symmetry, i.e. if it can be written in terms of the Casimir operators or generators of a given Lie algebra, then the solution of Eq. (3) is an element of the corresponding Lie group [4]. Consequently the two time influence functional of Eq. (8) is simply a diagonal group matrix element for the lowest-weight state and it can be evaluated using standard group-theoretical methods. 
If the Interacting Boson Model is used to describe the effects of nuclear structure on fusion, $H_{0}=H_{\mathrm{IBM}}$, and there are two choices for $H_{\mathrm{int}}$. One possibility is to take $H_{\text {int }}$ to be of the form of the most general one-body transition operator for IBM

$$
H_{\mathrm{int}}=V(r)+\sum_{k j l} \alpha_{k j l}(r)\left[b_{j}^{\dagger} \tilde{b}_{l}\right]^{(k)} \cdot Y^{(k)}(\hat{\mathbf{r}}),
$$

where $b_{j}^{\dagger}$ and $b_{l}$ denote the boson creation and annihilation operators $\left(b_{0}=s, b_{2}=d\right.$, etc). The $k$ sum runs over $k=2,4, \ldots 2 l_{\max }$, with $l_{\max }$ representing the maximum spin of boson operators. Odd values of $k$ are excluded as a consequence of the reflection symmetry of the nuclear shape and the $k=0$ term is already included in the bare potential $V(r)$. The form factors $\alpha_{k j l}(r)$ represent the spatial dependence of the coupling between the intrinsic and translational motions. The interaction term given in Eq. (9) is an element of the SU(6) algebra for the original form of the IBM with $s$ and $d$ bosons, and is an element of the $\mathrm{SU}(15)$ algebra when $g$ bosons are included as well [8].

To simplify the calculation of the influence functional, we can perform a rotation at each instant to a frame in which the $z$-axis points along the direction of relative motion. Neglecting the resulting centrifugal and Coriolis terms in this rotating frame is equivalent to ignoring the angular dependence of the original Hamiltonian. In this approximation, the coupling form factors become independent of $\ell$ and only $m=0$ magnetic sub-states of the target are excited [15]. For heavy systems, the neglected centrifugal and Coriolis forces are small. We take the scattering to be in the $x-y$ plane. Then making a rotation through the Euler angles $\hat{\mathbf{b}}=(\phi, \pi / 2,0)$, we can write the Hamiltonian as the rotation of a simpler Hamiltonian depending only on the magnitude of $\mathbf{r}$

$$
H=R(\hat{\mathbf{b}}) H^{(0)}(r) R^{\dagger}(\hat{\mathbf{b}}) .
$$

Since in Eq. (1) $H_{0}$ and $H_{k}$ are rotationally invariant, $H_{\text {int }}$ is the only term whose form is affected by this transformation. Hence we introduce the rotated interaction Hamiltonian $H_{\text {int }}^{(0)}(r)$, given by

$$
\begin{aligned}
H_{\mathrm{int}} & =R(\hat{\mathbf{b}}) H_{\mathrm{int}}^{(0)}(r) R^{\dagger}(\hat{\mathbf{b}}), \\
H_{\mathrm{int}}^{(0)}(r) & =\sum_{j l m} \phi_{j l m}(r) b_{j m}^{\dagger} b_{l m}, \\
\phi_{j l m}(r) & =(-)^{m} \sum_{k} \sqrt{\frac{2 k+1}{4 \pi}}\langle j m l-m \mid k 0\rangle \alpha_{k j l}(r) .
\end{aligned}
$$

If we assume now that the form factors $\alpha_{k j l}(r)$ are all proportional to the same function of $r$, then the Hamiltonian $H_{\mathrm{int}}^{(0)}$ commutes with itself at different times and hence we can write the two-time influence functional as

$$
\rho_{M}=\left\langle n_{i}\left|e^{i \int_{0}^{\tilde{T}} d t H_{\mathrm{int}}^{(0)}(\tilde{r}(t)) / \hbar} e^{-i \int_{0}^{T} d t H_{\mathrm{int}}^{(0)}(r(t)) / \hbar}\right| n_{i}\right\rangle .
$$

Since the exponents of the two operators in the influence functional commute, $\rho_{M}$ becomes the matrix element of an $\mathrm{SU}(6)$ transformation between $\mathrm{SU}(6)$ basis states, 
in other words it is a representation matrix element for this group and can be easily calculated using standard techniques. The two-time influence functional for the $s d-$ version of IBM was calculated in Ref. [11] and, for the particular case of the $\mathrm{SU}(3)$ limit, in Ref. [9].

The above method corresponds to a linear coupling approximation. Due to the exponential nature of fusion cross sections, it is not clear at the outset whether one can include the effects of the higher order terms in $H_{\text {int }}$ through a renormalization of the coupling strength. We next discuss an alternative method, whereby one can include the effects of coupling to all orders in the interaction Hamiltonian. In this case the solution of Eq. (3) is no longer an element of the appropriate group. Nevertheless, we can exploit the symmetry properties of the resolvent operator directly without utilizing its path integral representation. Such a Green's function approach has also been used to study quantum tunneling in a heat bath [16].

To include the effects of couplings to all orders, the interaction Hamiltonian in Eq. (1) is written as

$$
H_{\text {int }}(\mathbf{r}, \xi)=V_{\text {Coul }}(\mathbf{r}, \xi)+V_{\text {nuc }}(\mathbf{r}, \xi) .
$$

Here the Coulomb part is

$$
\begin{aligned}
V_{\text {Coul }}(\mathbf{r}, \xi) & =\frac{Z_{1} Z_{2} e^{2}}{r}\left(1+\frac{3}{5} \frac{R_{1}^{2}}{r^{2}} \hat{O}\right) \quad\left(r>R_{1}\right), \\
& =\frac{Z_{1} Z_{2} e^{2}}{r}\left(1+\frac{3}{5} \frac{r^{2}}{R_{1}^{2}} \hat{O}\right) \quad\left(r<R_{1}\right),
\end{aligned}
$$

and the nuclear part is taken to have the Woods-Saxon form,

$$
V_{\text {nuc }}(\mathbf{r}, \xi)=-V_{0}\left(1+\exp \frac{r-R_{0}-R_{1} \hat{O}(\hat{\mathbf{r}}, \xi)}{a}\right)^{-1}
$$

In Eqs. (16) and (17), $R_{0}$ is the sum of the target and projectile radii and $R_{1}$ is the mean radius of the deformed target. $\hat{O}$ is a general coupling operator between the internal coordinates and the relative motion which we take to be

$$
\hat{O}(\hat{\mathbf{r}}, \xi)=\sum_{k} v_{k} T^{(k)}(\xi) \cdot Y^{(k)}(\hat{\mathbf{r}}) .
$$

The $v_{k}$ represent the strengths of the various multipole transitions in the target nucleus. In the standard IBM with $s$ and $d$ bosons, the possible transition operators have $k=2,4 ; k=0$ and odd- $k$ values being excluded for reasons stated after Eq. (9). The quadrupole and hexadecapole operators are given by

$$
\begin{aligned}
T^{(2)} & =\left[s^{\dagger} \tilde{d}+d^{\dagger} s\right]^{(2)}+\chi\left[d^{\dagger} \tilde{d}\right]^{(2)}, \\
T^{(4)} & =\left[d^{\dagger} \tilde{d}\right]^{(4)} .
\end{aligned}
$$

We adopt the "consistent-Q" formalism of Casten and Warner [17], in which $\chi$ in Eq. (19) is taken to be the same as in $H_{\text {IBM }}$ (fitted to reproduce the energy level scheme and the electromagnetic transition rates of the target nucleus), and thus is not a free parameter. 
To calculate the fusion cross section to all orders, we consider the resolvent operator for the system

$$
G^{+}(E)=\frac{1}{E^{+}-H_{k}-H_{\mathrm{IBM}}(\xi)-H_{\mathrm{int}}(r, \hat{O})} .
$$

The basic idea is to find the interaction representation which diagonalizes $H_{\text {int }}$. Note that, in general, it is not possible to diagonalize $H_{\mathrm{IBM}}$ and $H_{\text {int }}$ simultaneously, so the effect of the excitation energies in the target nucleus are ignored in this approach. To this end, we need to identify the unitary transformation which diagonalizes the operator $\hat{O}$

$$
\hat{O}_{d}=\mathcal{U} \hat{O} \mathcal{U}^{\dagger},
$$

so that we can calculate its eigenvalues and eigenfunctions

$$
\hat{O}_{d}|n\rangle=h_{n}|n\rangle .
$$

Assuming the completeness of these eigenfunctions

$$
\sum_{n}|n\rangle\langle n|=1,
$$

one can write the matrix element of the resolvent as

$$
\begin{aligned}
\left\langle\xi_{f}, r_{f}\left|G^{+}(E)\right| \xi_{i}, r_{i}\right\rangle & =\left\langle\xi_{f}, r_{f}\left|\mathcal{U}^{\dagger} \mathcal{U}\left[E^{+}-H_{k}-H_{\mathrm{int}}(r, \hat{O})\right]^{-1} \mathcal{U}^{\dagger} \mathcal{U}\right| \xi_{i}, r_{i}\right\rangle, \\
& =\left\langle\xi_{f}, r_{f}\left|\mathcal{U}^{\dagger}\left[E^{+}-H_{k}-H_{\mathrm{int}}\left(r, \hat{O}_{d}\right)\right]^{-1} \sum_{n}\right| n\right\rangle\left\langle n|\mathcal{U}| \xi_{i}, r_{i}\right\rangle, \\
& =\sum_{n}\left\langle\xi_{f}\left|\mathcal{U}^{\dagger}\right| n\right\rangle\left\langle n|\mathcal{U}| \xi_{i}\right\rangle\left\langle r_{f}\left|G_{n}^{+}\right| r_{i}\right\rangle,
\end{aligned}
$$

where

$$
G_{n}^{+}(E)=\frac{1}{E^{+}-H_{k}-H_{\text {int }}\left(r, h_{n}\right)} .
$$

$G_{n}^{+}(E)$ in Eq. (26) is the resolvent operator for one-dimensional motion in the potential $H_{\text {int }}\left(r, h_{n}\right)$, whose fusion cross section can be easily calculated within the standard WKB approximation. The total cross section follows from multiplying these eigenchannel cross sections by the weight factors indicated in Eq. (25). The calculation of the weight factors $\left\langle n|\mathcal{U}| \xi_{i}\right\rangle$ within the IBM is straightforward and is given in Refs. [12, 14], to which the reader is referred for further details.

Comparison of the linear and all orders coupling calculations shows that both can describe fusion cross sections equally well. However there are important differences between the two approaches when one considers barrier distributions, and only through the latter, can one obtain a good representation of the data [12]. Another advantage of the all orders coupling is that the strengths used are very close to the deformation parameters and it is easier to establish a contact with the geometrical model. (In contrast, rather large strengths are required in the linear coupling approximation.) 


\section{A Systematic Study of Subbarrier Fusion in Rare- Earth Nuclei}

We carried out a systematic study of subbarrier fusion data, accumulated over the last few years, for rare-earth nuclei [13]. To illustrate the quality of this global fit, in Fig. 1 we compare our theoretical result for the ${ }^{16} \mathrm{O}+{ }^{148} \mathrm{Sm},{ }^{16} \mathrm{O}+{ }^{154} \mathrm{Sm}$, and ${ }^{16} \mathrm{O}+{ }^{186} \mathrm{~W}$ systems with the data from the Australian National University. There is excellent agreement between the IBM-based model and the data, as the target nucleus changes from vibrational $\left({ }^{148} \mathrm{Sm}\right)$ to deformed with positive $\left({ }^{154} \mathrm{Sm}\right)$ and negative $\left({ }^{186} \mathrm{~W}\right)$ hexadecapole moments.

Having extracted a consistent set of parameters, we can predict fusion cross sections and barrier distributions in other rare-earth nuclei. In this respect, the transitional Os-Pt region is of particular interest. One expects that barrier distributions for transitional nuclei exhibit sharp changes due to shape-phase transitions, contrary to vibrational and rotational nuclei where the cross sections increase smoothly with increasing deformation or mass number [13]. An accurate measurement of subbarrier fusion cross sections for transitional nuclei such as $\mathrm{Pt}$ and Os provides a sensitive test for competing models in this region and possibly point to new directions in research. A subsequent measurement of the fusion cross sections for ${ }^{40} \mathrm{C}++{ }^{194} \mathrm{Pt}$ and ${ }^{40} \mathrm{C}++{ }^{194}$ Os systems confirmed our predictions 21 .

It is also possible to generalize the previous formalism to include arbitrary kinds of bosons in the target nucleus and investigate whether $g$ bosons have any discernible effects on subbarrier fusion reactions. We found that [14 except for slight differences in the barrier distributions (which can be made even smaller by fine tuning the coupling strengths), there are no visible differences between the $s d$ and $s d g$ model predictions. The similarity of the results implies that subbarrier fusion probes the overall coupling strength in nuclei, but otherwise is not sensitive to the details of the nuclear wavefunctions. In this sense, subbarrier fusion is in the same category as other static quantities (energy levels, electromagnetic transition rates), and does not seem to constitute a dynamic probe of nuclei, in contrast to proton scattering [18].

Another experimental test of our model would be to study angular momentum distributions in subbarrier fusion, which can be determined reasonably accurately from the gamma-ray multiplicities data or from the measurement of relative populations of the ground and isomeric states in evaporation residues [6]. Vandenbosch and his collaborators at the University of Washington, Seattle devoted considerable time to the measurement of angular momentum distributions [6, 19]. In Fig. 2, we present angular momentum distributions calculated using our model for a number of systems 20]. We should emphasize that these angular momentum distributions are not direct experimental observables. However there are relations of rather general nature relating angular momentum distributions to the moments of fusion cross sections [22] which can be used to assess the consistency of the direct measurements of the fusion cross sections with the angular momentum distributions extracted from gamma-ray multiplicities.

We would like express our gratitude to our collaborators, J. Bennett, A.J. De- 
Weerd, and N. Takigawa, without whom the work reported here would not be possible. This research was supported in part by the U.S. National Science Foundation

Grants No. PHY-9605140 and INT-9315876, in part by the University of Wisconsin Research Committee with funds provided by the Wisconsin Alumni Research Foundation, and in part by grants from the Australian Research Council and the Department of Industry, Science and Technology.

\section{References}

[1] S.G. Steadman and M.J. Rhoades-Brown, Ann. Rev. Nucl. Sci. 36, 649 (1986); M. Beckerman, Rep. Prog. Phys. 51, 1047 (1988).

[2] A.B. Balantekin and N. Takigawa, Rev. Mod. Phys., October 1997.

[3] N. Rowley, G.R. Satchler, and P.H. Stelson, Phys. Lett. B254, 25 (1991).

[4] A.B. Balantekin and N. Takigawa, Ann. Phys. (NY) 160, 441 (1985).

[5] H. Esbensen, Nucl. Phys. A352, 147 (1981).

[6] R. Vandenbosch, Ann. Rev. Part. Nucl. Sci. 42, 447 (1992).

[7] J.X. Wei et al., Phys. Rev. Lett. 67, 3368 (1991); J.R. Leigh et al., Phys. Rev. C47, R437 (1993).

[8] F. Iachello and A. Arima, The Interacting Boson Model, (Cambridge, 1987).

[9] A.B. Balantekin, J. Bennett and N. Takigawa, Phys. Rev. C44, 145 (1991).

[10] S. Kuyucak and I. Morrison, Ann. Phys. (NY) 181, 79 (1988); 195, 126 (1989).

[11] A.B. Balantekin, J.R. Bennett, A.J. DeWeerd, and S. Kuyucak, Phys. Rev. C46 (1992) 2019.

[12] A.B. Balantekin, J.R. Bennett, and S. Kuyucak, Phys. Rev. C48, 1269 (1993).

[13] A.B. Balantekin, J.R. Bennett, and S. Kuyucak, Phys. Rev. C49, 1079 (1994).

[14] A.B. Balantekin, J.R. Bennett, and S. Kuyucak, Phys. Rev. C49, 1294 (1994).

[15] N. Takigawa and K. Ikeda, Proc. Symp. on Many Facets of Heavy Ion Fusion Reactions, Ed. W. Henning et al., (Argonne : ANL-PHY-86-1) (1986), pp. 613620; H. Esbensen, S. Landowne, and C. Price, Phys. Rev. C36, 1216 (1987); O. Tanimura, Phys. Rev. C35, 1600 (1987).

[16] N. Takigawa, Y. Alhassid, and A.B. Balantekin, Phys. Rev. C45, 1850 (1992); A.B. Balantekin, J.R. Bennett, N. Takigawa, and Y. Alhassid, Japanese J. Appl. Phys. Series 9, 90 (1993). 
[17] R.F. Casten and D.D. Warner, Rev. Mod. Phys. 60, 389 (1988).

[18] S. Kuyucak and I. Morrison, Phys. Rev. C48, 774 (1993).

[19] S. Gil et al, Phys. Rev. C43, 701 (1991); Phys. Rev. Lett. 65, 3100 (1990).

[20] A.B. Balantekin, J.R. Bennett, and S. Kuyucak, Phys. Lett. B335, 295 (1994) (nucl-th 9407037).

[21] J.D. Bierman et al., Phys. Rev. Lett. 76, 1587 (1996).

[22] A.B. Balantekin, A.J. DeWeerd, and S. Kuyucak, Phys. Rev. C 54, 1853 (1996).

\section{Figure Captions}

Fig. 1. Comparison of fusion cross sections and barrier distributions predicted using the Interacting Boson Model with the data as described in the text. The parameters are given in Ref. 13.

Fig. 2. Comparison of angular momentum distributions predicted using the Interacting Boson Model with the data. The parameters are given in Ref. [20]. 